\title{
Numerical Simulations of a Single Injector Gaseous Methane Rocket Combustion Chamber
}

\author{
Roman Keller* and Peter Gerlinger ${ }^{\dagger}$ \\ Institute of Combustion Technology for Aerospace Engineering \\ University of Stuttgart, 70569 Stuttgart, Germany
}

\begin{abstract}
Numerical simulations of a single injector gaseous methane/oxygen rocket combustion chamber from the Technische Universität München at 20 bar pressure are performed using steady RANS techniques on axisymmetric and three-dimensional grids. In a first step the combustion chamber with a square cross-section is simulated using a two-dimensional grid with the assumptions of axisymmetry. This setup is used for a comprehensive parameter study. In a next step a three-dimensional simulation using the findings from the parameter study is performed. It is shown that the $q-\omega$ and SST turbulence model produce comparable results and that the turbulent Schmidt and Prandtl numbers have a significant quantitative influence. The presented flow field results indicate that three-dimensional effects are important, questioning the validity of axisymmetry for this chamber. Both the two-dimensional simulations, despite the axisymmetry assumption, and the three-dimensional simulation show good agreement with the experimental values for the wall heat flux and the wall pressure.
\end{abstract}

\section{Introduction}

For the numerical simulation of rocket combustion chambers the main aspects are reliable and precise predictions of the flow field, the location of the flame, pressure oscillations, and the thermal wall heat load. Up to now experimental investigations ${ }^{1,2}$ are the most trusted approach in rocket combustor development. However, due to the high pressure levels even model combustor tests are very expensive and the exact replication of flight conditions in ground test facilities is difficult to achieve. Another disadvantage is the lack of available data from the interior of the combustor due to the challenges for optical diagnostics to be used in high pressure environments. ${ }^{3}$ In comparison to experiments numerical simulations have the advantage that a complete data set is obtained. But the complex processes in rocket combustors require further research in several fields. In addition, the number of available experimental test cases for code validation is very low. Most of these experiments offer limited data only (e.g. wall pressures, temperatures, and wall heat fluxes) ${ }^{1,4,5}$ but rarely comprehensive information about velocity, species and flame properties as they are known for low pressure laboratory flames. ${ }^{6}$ Up to now, most scientific simulations of rocket combustors are performed for simple geometries only (e.g. single coaxial injectors) which strongly differ from full scale engines. Moreover, large discrepancies are observed between different numerical techniques employed for the same test case $^{7,8}$ and the reasons for these deviations are often unknown. There is no common opinion whether rocket combustion chambers can be simulated using steady state techniques (RANS) ${ }^{8,9}$ or if unsteady methods are required (URANS/LES). ${ }^{10-12}$

In this paper a methane rocket combustion chamber is studies which has been investigated experimentally at the Technische Universität München. In comparison to the PennState Perburner Combustor ${ }^{1,4}$ where only wall heat flux data is available for comparison with numerical results, the experimental measurements also include wall pressures which are used as a second validation parameter. This second validation parameter may help to better benchmark different simulations. The chamber has a square cross section but axisymmetric as well as three-dimensional simulations are performed. Although the axisymmetry assumptions are inaccurate, the lower resources required for the two-dimensional simulations offer large advantages. Using the less

*PhD Student, Computer Simulation.

${ }^{\dagger}$ Adjunct Professor, Computer Simulation. 
expensive setups influence parameters can be tested much faster and thus a much broader spectrum of them can be simulated. Following matters are of special interest in this study:

1. influence of the turbulence model (here: $\mathrm{q}-\mathrm{w}$ and SST) on the flow field and the wall heat flux,

2. influence of the turbulent Prandtl and Schmidt numbers,

3. viability of the axisymmetric oversimplification, and

4. the capability of RANS simulations to predict the occurring flow features correctly.

These findings are then used for the setup of the three-dimensional simulation. The paper is organized in the following way. In the next section the numerical method is briefly explained. Then the test case is introduced, followed by the results of the two- and three-dimensional simulations. At the end a brief conclusion is given.

\section{Numerical Method}

The simulations are performed with the in-house CFD code TASCOM3D (Turbulent All-Speed Combustion Multigrid). TASCOM3D has been used for simulations of reacting ${ }^{13-17}$ and non-reacting ${ }^{18-21}$ sub- and supersonic flows as well as rocket combustion chamber simulations. ${ }^{10,11}$ The three-dimensional compressible Navier-Stokes equations are

$$
\frac{\partial \mathbf{Q}}{\partial t}+\frac{\partial\left(\mathbf{F}-\mathbf{F}_{\nu}\right)}{\partial x}+\frac{\partial\left(\mathbf{G}-\mathbf{G}_{\nu}\right)}{\partial y}+\frac{\partial\left(\mathbf{H}-\mathbf{H}_{\nu}\right)}{\partial z}=\mathbf{S}
$$

where $t$ is the physical time and $Q$ is the conservative variable vector given by

$$
\mathbf{Q}=\left[\bar{\rho}, \bar{\rho} \tilde{u}, \bar{\rho} \tilde{v}, \bar{\rho} \tilde{w}, \bar{\rho} \tilde{E}, \bar{\rho} k, \bar{\rho} \omega, \bar{\rho} \tilde{Y}_{i}\right]^{t}, \quad i=1,2, \ldots, N_{k}-1 .
$$

The inviscid fluxes are represented by $\mathbf{F}, \mathbf{G}$ and $\mathbf{H}$ in $x$-, $y$-, and $z$-direction, respectively, and the corresponding viscous fluxes are denoted by the index $\nu$. The variables in the variable vector $\mathbf{Q}$ are the Reynolds-averaged density $\bar{\rho}$, the Favre-averaged velocities $\tilde{u}, \tilde{v}$, and $\tilde{w}$, and the total specific energy $\tilde{E}$. The $k$ and $\omega$ variables represent the turbulent energy and turbulent frequency $(\omega=\epsilon / k$ where $\epsilon$ is dissipation rate of $k$ ) for the two equations turbulence models. The two different models used are the $q-\omega$ model by Coakley and Huang ${ }^{22}$ and the SST (shear stress transport) model by Menter. ${ }^{23}$ Finally $\tilde{Y}_{i}$ are species mass fractions and $N_{k}$ is the number of species considered. The source vector

$$
\mathbf{S}=\left[0,0,0,0,0, S_{k}, S_{\omega}, \bar{S}_{Y_{i}}\right]^{t}, \quad i=1,2, \ldots, N_{k}-1
$$

contains entries from turbulence and chemical reactions.

The governing equations are solved with an implicit finite-volume scheme. The solver works on structured multi-block grids using a Lower-Upper Symmetric Gauss-Seidel (LU-SGS) ${ }^{14,24,25}$ algorithm and is fully parallelized and vectorized. It shows an excellent performance on vector computers as well as on massively parallel architectures. ${ }^{26}$ To calculate the inviscid fluxes the $\mathrm{AUSM}^{+}$-up flux vector splitting of $\mathrm{Liou}^{27}$ is used. This requires primitive variables at the cell interfaces of the structured grid which are determined by a $5^{\text {th }}$ order upwind biased MLPld scheme. $^{21}$ The viscous fluxes are calculated by central differences. The combustion is modeled using finite-rate chemistry and is treated implicitly and fully coupled with the fluid field. A reaction mechanism with 21 species and 98 reactions specially designed for the investigated combustor conditions ${ }^{28}$ is used for the calculations.

In case of steady-state simulations the solution is advanced in time using local time stepping with a constant CFL number.

\section{Experimental Setup}

The simulated methane rocket combustion chamber is experimentally investigated at the Technische Universität München (TUM). ${ }^{29,30}$ The model chamber is designed to be used as a validation test case for high pressure methane rocket combustion simulations. 
The chamber has a length of $290 \mathrm{~mm}$ and a square cross section with an edge length of $12 \mathrm{~mm}$. Gaseous oxygen and methane are injected into the chamber through a single shear coaxial injector. Oxygen is fed to the chamber through the inner tube of the injector, which has a diameter if $4 \mathrm{~mm}$. The annular methane feed has an inner and outer diameter of $5 \mathrm{~mm}$ and $6 \mathrm{~mm}$, respectively. The nozzle is two-dimensional with a contraction ratio of 2.5. There are several operation points available for the simulation ${ }^{29}$ but in this paper, only one operation point (described below) is simulated. Oxygen and methane are injected at $278 \mathrm{~K}$ and $269 \mathrm{~K}$ with a mass flow rate of $45 \mathrm{~g} / \mathrm{s}$ and $17 \mathrm{~g} / \mathrm{s}$, respectively. This leads to an oxidizer-to-fuel ratio $(\mathrm{O} / \mathrm{F})$ of 2.62 and an overall mass flow rate of $62 \mathrm{~g} / \mathrm{s}$. The chamber is operated at a nominal pressure of 20 bar.

Due to the capacitive cooling of the chamber only short test runtimes are possible. From the transient measurement values, the boundary conditions for the simulation are extracted during a short time period and a quasi-steady state is assumed. The experimental data include the axial distribution of the wall temperatures, the wall heat fluxes, and the wall pressure in the chamber.

\section{Numerical Setup}

Two-dimensional axisymmetric and three-dimensional simulations with steady simulation techniques are performed for this paper. The entire model combustion chamber is simulated together with the adjacent convergent-divergent nozzle and the injector. The inclusion of the convergent-divergent nozzle with its supersonic outflow has the advantage that no pressure forcing boundary condition has to be used. Instead, the chamber pressure is determined by the mass flow through the nozzle throat. The supersonic part of the nozzle is shortened to save computational time. The coaxial injector for both methane and oxygen is simulated with a length of $29 \mathrm{~mm}$.

The boundary conditions are set according to the test case descriptions. ${ }^{29}$ The combustion chamber walls are set to isothermal, no-slip walls. The wall temperatures are taken from the experimental measurements (axial profile) ${ }^{29}$ and assumed to be constant in circumferential direction. The vertical wall (faceplate) and the nozzle wall temperatures are extrapolated from the experimental values since no measured data is available there. A supersonic outflow boundary condition is used at the diverging nozzle exit. The injector walls are simulated with adiabatic, no-slip wall boundary conditions. At the injector inflow, mass-flow boundary conditions are utilized which regulate the incoming mass-flow by varying the averaged inflow velocity. The inflow profiles are extracted from separate calculations of the entire injector feed system up to the porous plates used in the experiment. ${ }^{29}$ This is necessary because the inflow conditions at the injector have a significant influence on the flow field in the combustion chamber. The computational grid is refined towards the walls down to a normal cell height of $10^{-6} \mathrm{~m}$ to achieve $y^{+} \simeq 1$, except for the vertical wall (faceplate) where a cell size of $5 \cdot 10^{-6} \mathrm{~m}$ is chosen. All simulation are run using the $5^{\text {th }}$ order upwind biased MLPld discretization. The turbulent Prandtl and Schmidt numbers are set to 0.9 and 0.7 in all simulations except in Section V.C where a parameter study on the turbulent Prandtl and Schmidt numbers is performed.

\section{IV.A. Two-dimensional steady simulations (2D-RANS)}

The two-dimensional grid for the steady simulations consists of about 38 thousand cells. The chamber has 224 cells in $x$-direction and 160 cells in $y$-direction. The oxygen and methane injector as well as the post tip are resolved with 32 cells each. Figure 1 a) shows the two-dimensional grid. Please note that only every second cell is shown and the grid is not to scale but strongly stretched in $y$-direction.

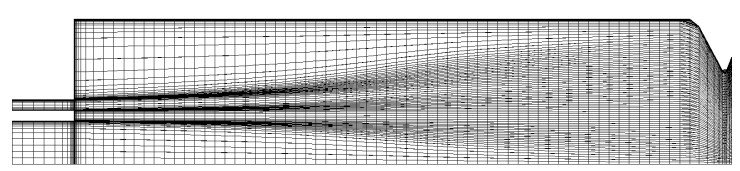

(a) 2D grid

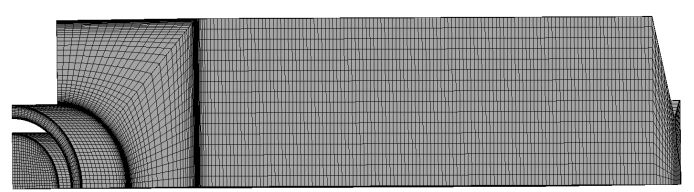

(b) 3D grid.

Figure 1. Computational grids with every second cell drawn. 


\section{IV.B. Three-dimensional steady simulations (3D-RANS)}

The domain of the three-dimensional RANS simulation consists of a quarter of the combustion chamber, using symmetry boundary conditions at the symmetry planes $(\mathrm{z}=0$ and $\mathrm{y}=0)$. The quarter of the model chamber is discretized with 836 thousand cells. The blocks around the symmetry axis are constructed with a butterfly grid to fit the round geometry into a structured multi-block grid. The axial resolution is 160 cells. In $y$-direction 160 cells are used. The number of cell volumes in the azimuthal direction is 32 . The oxygen and methane injector as well as the post tip are resolved with 32 cells each. Figure $1 \mathrm{~b}$ ) shows the three-dimensional grid. Please note that only every second cell is shown and the grid is not to scale but strongly stretched in $y$ - and $z$-direction.

\section{Results}

Results from previous simulations ${ }^{10,11}$ and other groups $s^{7,12,31,32}$ suggest that steady simulation cannot correctly model all the occurring features of the strongly unsteady flow field in single injector rocket combustion chambers. Due to the finer computational grids and the required averaging of the solution, unsteady simulations are not feasible for most industrial applications. Work from other researches showed that steady simulations can achieve a good agreement with the experimental results. ${ }^{8}{ }^{9}$ In the present work, RANS simulations are presented. This is done for two reasons. First, solutions can be achieved much faster. This renders extensive parameter studies on the influence of e.g. inflow conditions, grid dependencies, or turbulence models possible. It is hoped, that these findings can later be used on more complex simulations. Second, the predictive capabilities of RANS simulations for this test case in particular are investigated. If steady-state solutions are capable of achieving good agreement with the experimental data this could be due to:

- the different gaseous fuel used (methane instead of hydrogen),

- the lower impulse ration of the coaxial injector, or

- the lower expansion ratio from the injector to the chamber which decreases the size of the recirculation zone,

compared to other single injector gaseous test chambers (e.g. PennState Preburner Combustor).

\section{V.A. Diameter selection for two-dimensional simulations}

Since the chamber is not axisymmetric but has a square cross section, an appropriate diameter for the axisymmetric simulation must be selected. Three different diameters for the chamber with the same injector and nozzle geometry are tested and the most promising is used for the following investigations. All these three diameters preserve an important feature of the original square cross section. The three diameters are sketched in Fig. 2. The throat area is kept constant for all simulations.

1. Diameter $1(12 \mathrm{~mm})$ corresponds to the smallest distance from the injector to the wall. It achieves an identical hydraulic diameter (ratio between area and circumference) as the square cross section of the chamber. Thus the ratio between volume and surface area of the chamber is preserved.

2. A circle with diameter $2(13.5 \mathrm{~mm})$ has the same area as the square cross section. This configuration preserves the expansion ratio between the injector and the chamber area. This will result in the most similar injector dominated

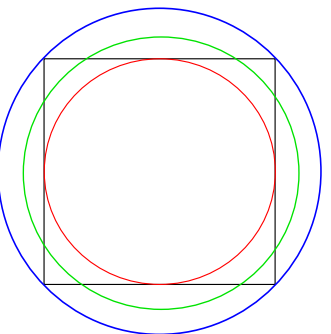

Figure 2. Square cross section of the combustion chamber (black), diameter 1 (red), diameter 2 (green), and diameter 3 (blue). flow field with comparable Mach numbers and average velocities.

3. Diameter $3(17 \mathrm{~mm})$ corresponds to the largest wall distance from the injector. Since the recirculation zones are expected to be strongest in the corners, this will presumably resemble the recirculation dominated flow field most adequately.

Figure 3 shows the temperature contours and the recirculation zone for the two-dimensional simulations with the different diameters. All simulations show a very thin flame in the first half of the chamber. The flames 
temperature, K $500 \quad 1000 \quad 1500 \quad 2000 \quad 2500 \quad 3000$
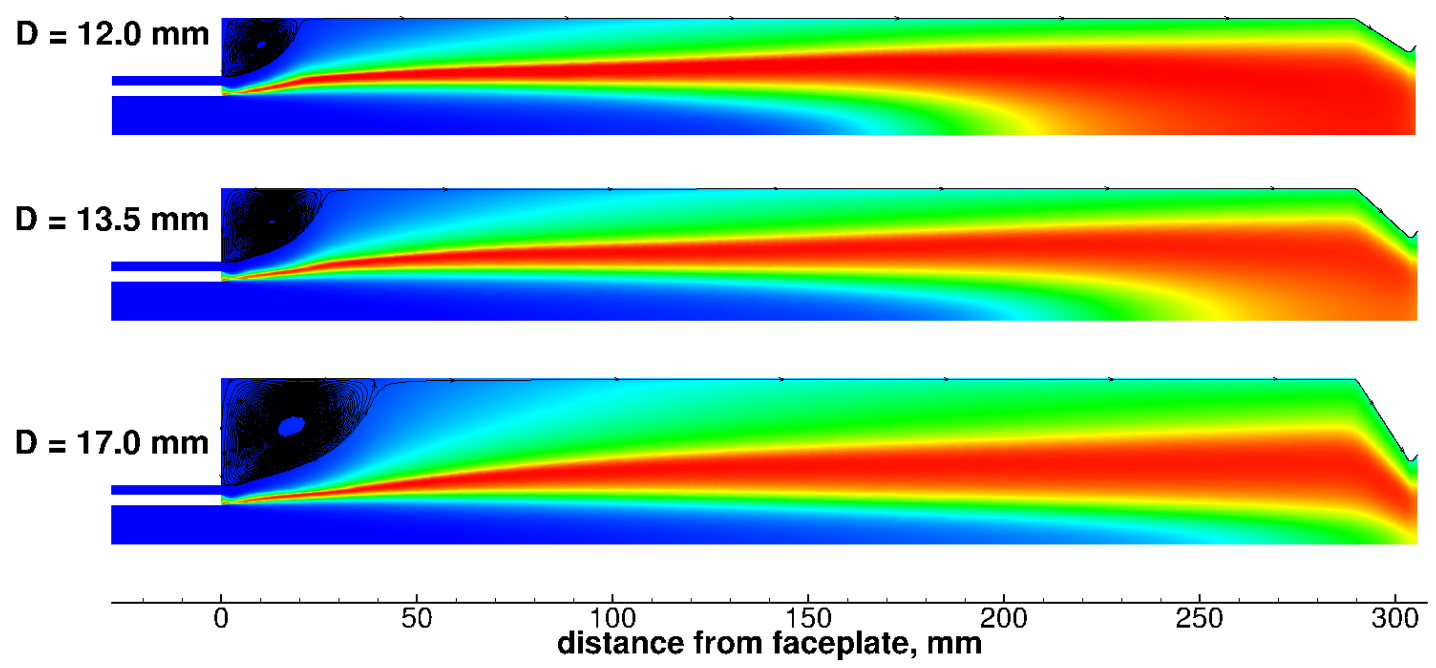

Figure 3. Temperature contour plots for RANS simulations with different diameters. $\mathrm{D}=12.0 \mathrm{~mm}($ top $)$, D $=13.5$ mm (middle), and $\mathrm{D}=17.0 \mathrm{~mm}$ (bottom).

thickening towards the end of the chamber is amplified by a smaller diameter. Because the high temperature region is further away from the wall with increasing diameter, a lower temperature gradient at the wall can be presumed which will decrease the expected wall heat flux. Due to the increasing expansion ration between the injector and the chamber the recirculation zones grow in height and length with increasing diameter.

Figure 4 shows the oxygen and methane mass fraction distribution in the chamber. Since the high concentrations of both substances are separated by the burning zone, they can be plotted in one picture. The oxygen is mainly distributed along the symmetry axis and the pure methane stays mainly at the top left corner in the recirculation zone. The length of the oxygen jet increases with the diameter of the chamber. This is in good agreement with the increased temperature at the center line in Fig. 3.

Figure 5 a) shows the wall heat flux profiles from the different simulations and the experimental values. Additionally, the averaged heat flux values, scaled by surface area, are delineated as well. While diameter 3 results in a lower wall heat flux profile compared to diameter 1 and 2 , the qualitative agreement is the best with almost the same slope of the wall heat profile. Both diameter 1 and 2 overestimate the wall heat fluxes at the end of the chamber. The simulations show an initial peak in the wall heat flux distribution, which is located at the reattachment point of the recirculation zone. The position of the initial peak is consistent with the different sizes of the recirculation zone (see. Fig 3). The simulation with diameter 2 (13.5 mm) shows the best quantitative agreement for the profile and the averaged wall heat flux.

Figure $5 \mathrm{~b}$ ) depicts the pressure profiles (solid line) and the corresponding averaged pressure for the different diameters. Diameter 1 significantly overpredicts the pressure drop and the averaged pressure in the chamber. The simulation with diameter 3 has the smallest pressure decline and averaged pressure level of all simulations and underpredicts the measured values. Diameter 2 shows the best agreement with the experimental data for both the pressure profile and the averaged chamber pressure. While the simulations show large differences in the pressure profiles, the averaged pressure values are all within 0.5 bar. Overall, the simulation with diameter $2(13.5 \mathrm{~mm})$ achieves the best results. Thus it is chosen for all subsequent two-dimensional simulations.

\section{V.B. Influence of the turbulence model}

In this section, the turbulence model influence on the flow field, pressure, and wall heat flux is investigated. The turbulence model can have significant influence on rocket combustor results. ${ }^{8}$ The study is performed for two-dimensional steady RANS simulations. Results of simulations with the $q$ - $\omega$ and SST turbulence model 


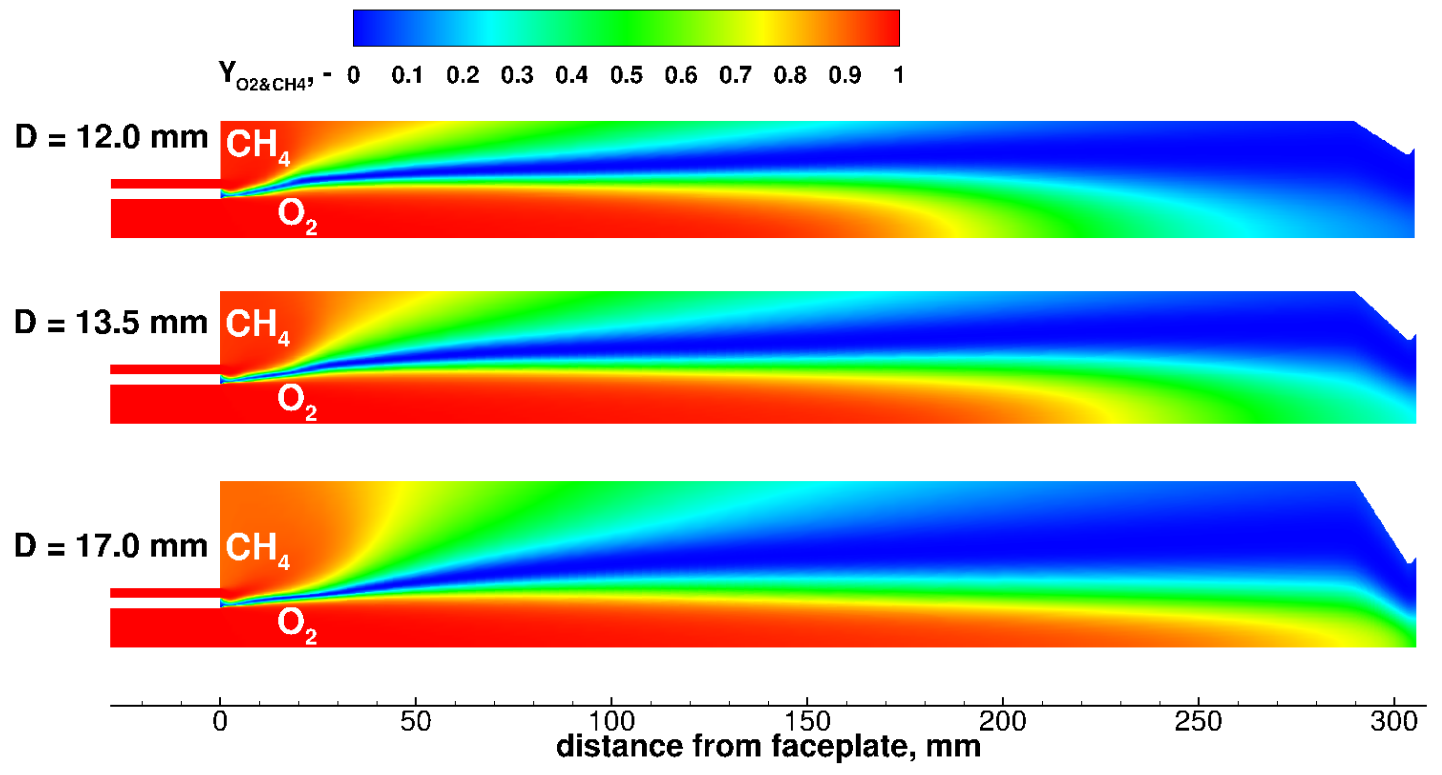

Figure 4. Oxygen and methane mass fraction contour plots for RANS simulations with different diameters. $\mathrm{D}=12.0$ $\mathrm{mm}$ (top), $\mathrm{D}=13.5 \mathrm{~mm}$ (middle), and $\mathrm{D}=17.0 \mathrm{~mm}$ (bottom).

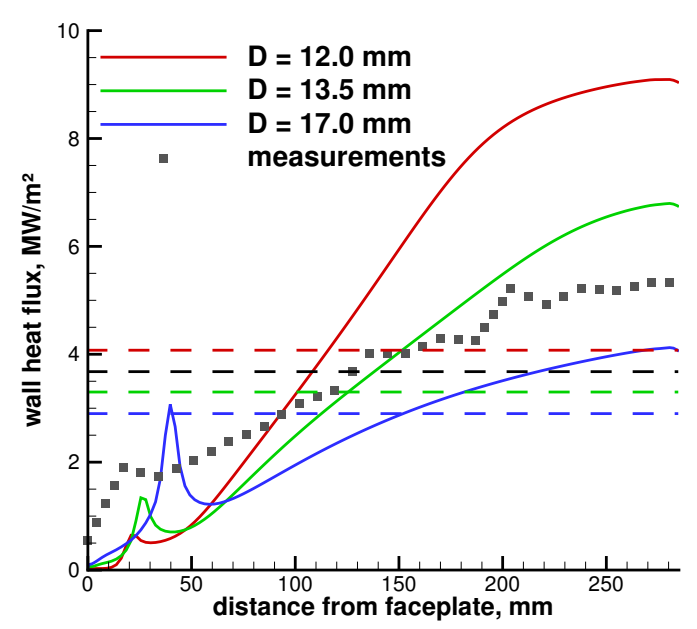

(a) Wall heat flux profiles.

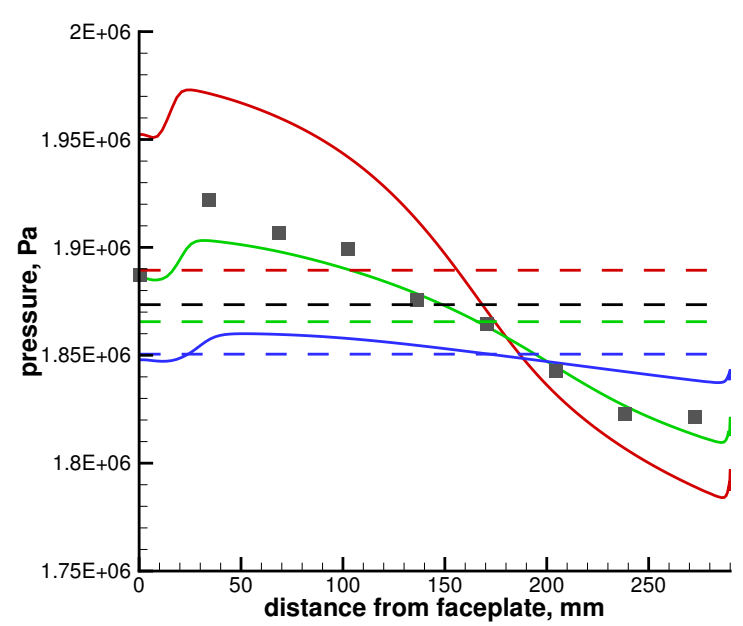

(b) Pressure profiles.

Figure 5. Influence of the diameter of the $2 \mathrm{D}$ chamber on the wall heat flux and pressure profiles. 
are compared.

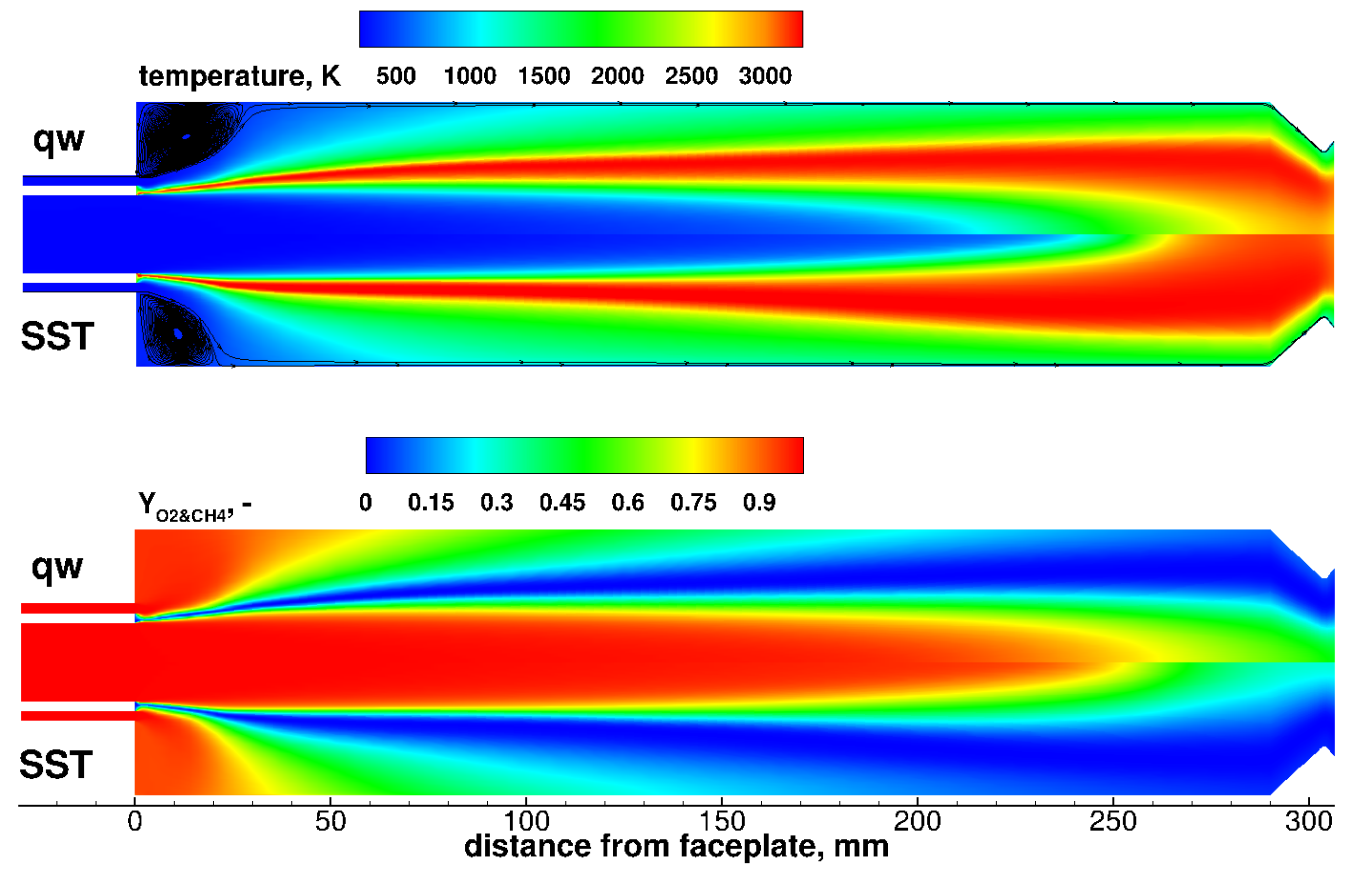

Figure 6. Temperature (top), oxygen and methane mass fraction (bottom) contour plots for RANS simulations with the $q-\omega$ (upper half) and SST (lower half) turbulence model.

In Fig. 6 the temperature contours (top) and the mass fraction distributions of oxygen and methane (bottom) for the $q-\omega$ (upper half) and SST turbulence model (lower half) are shown. Again, due to the separation of the high methane and oxygen concentrations by the combustion zone both can be depicted in one plot. Both turbulence models show similar temperature distributions. A major differences is the earlier temperature increase towards the center line at the end of the chamber in the simulation with the SST model. The $q-\omega$ model predicts a larger recirculation zone with a length of $27 \mathrm{~mm}$ compared to 21.5 $\mathrm{mm}$ in case of the SST model. Both simulations show similar species mass fraction distributions. The SST simulation has a lower oxygen concentration at the end of the chamber towards the nozzle. This is in good agreement with the higher temperature there.

Figure 7 a) shows the wall heat flux profiles for the simulations with the different turbulence models. For both simulations the maximal heat fluxes at the end of the combustor are nearly the same and the measured value is overpredicted by approx. $25 \%$. The measured heat flux profile rises steeply at the beginning of the chamber up to a local peak at approx. $17 \mathrm{~mm}$ downstream of the faceplate and shows a constant increase up to approx. $200 \mathrm{~mm}$. Afterwards, the measured heat flux shows an almost constant value of $\sim 5.2 \mathrm{MW} / \mathrm{m}^{2}$. For both simulations, the trend of the wall heat flux is not reproduced correctly. It is assumed that this is due to the wrong prediction of the flame position and shape.

The pressure distribution along the $x$-axis is depicted in Fig. 7 b). Again, the simulations with $q-\omega$ and SST model show similar results. The quantitative pressure predictions are very close to the measured values and the pressure peak at the beginning of the chamber is reproduced. However, the pressure plateau at the end of the chamber is not apparent in neither of the two turbulence model simulations. This pressure plateau is interpreted as a sign for the end of the combustion zone. Thus a correct prediction of this plateau is an important indication for a correct flame shape.

The two turbulence models show noticeable differences in both the flow field and the experimental wall profiles. Nevertheless, a superior model cannot be identified. The $q-\omega$ model is used for subsequent steady RANS simulations.

\section{V.C. Influence of turbulent Prandtl and Schmidt numbers}

In rocket combustion chambers turbulent species and heat transport is very important. Thus the choice of the turbulent Prandtl and Schmidt numbers has a significant influence on the solution. Unfortunately, 


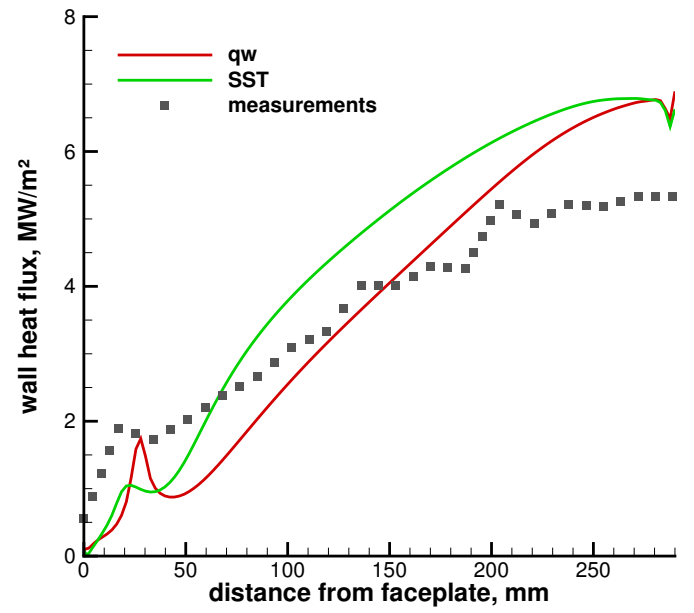

(a) Wall heat flux.

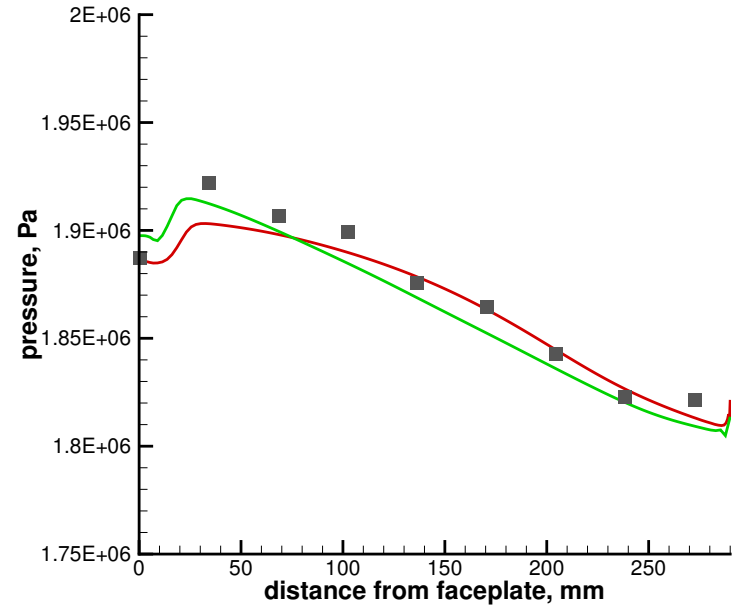

(b) Pressure.

Figure 7. Influence of the turbulence model on the wall heat flux and axial pressure distribution.

no reliable model for spatially and temporally varying turbulent Prandtl and Schmidt numbers is available. The effect of different, spatially constant turbulent Prandtl and Schmidt numbers is shown in this section. The reason for this investigation is not to find the best combination to fit the experimental profiles but to show their influence on the solution. A variation of the numbers between 0.7, 0.9, and 1.1 is performed.

Figure 8 shows temperature contours for different Prandtl and Schmidt numbers (0.7 and 0.9 only) using the q- $\omega$ turbulence model. The differences are mainly restricted to the center line at the end of the chamber. The effect of the turbulent Schmidt is more distinct for the temperature contours than the effect of the Prandtl number. The deviations due to the turbulent Schmidt number in the temperature contours are comparable to those due to the turbulence model.

Figure 9 a) depicts the wall heat fluxes for different Prandtl and Schmidt numbers. Each color shows a constant Prandtl number, while the Schmidt numbers are decoded by line patterns. With an increasing Prandtl number the wall heat flux decreases. For an increasing Schmidt number the wall heat flux increases slightly in the first third of the chamber and decreases in the rest of the chamber. While the Schmidt number changes the slope of the wall heat flux, the Prandtl number's effect is mainly a proportional factor. The shown variation does not improve the qualitative deficits discussed in Section V.B.

The chamber pressure, shown in Fig. 9 b) is also strongly influenced by both turbulent Prandtl and Schmidt number. The turbulent Schmidt number has a much larger effect. Again, the effect of turbulent Prandtl and Schmidt numbers is mainly on the averaged pressure and not on the slope or the qualitative characteristic of the pressure profile.

It is interesting to see that an increase in the wall heat flux and therefore an increase in energy that leaves the system, does not decrease the chamber pressure significantly. This is expected from a system energy point of view. ${ }^{10}$ Apparently, other effects besides the different energy losses at the wall must have an impact on the chamber pressure. One possible explanation for a higher pressure level together with higher wall heat fluxes is a higher combustion efficiency in the chamber. The combustion efficiency can be compared by the amount of unburnt reactants exiting the system. Table 1 shows the mass flow-weighted mass fraction of $\mathrm{O}_{2}, \mathrm{CH}_{4}, \mathrm{H}_{2} \mathrm{O}$, and $\mathrm{CO}_{2}$ in the nozzle throat for different turbulent Prandtl and Schmidt numbers. The mass fraction of the reactants $\left(\mathrm{O}_{2}\right.$ and $\left.\mathrm{CH}_{4}\right)$ are still relatively high. From equilibrium calculations with $\mathrm{CEA}^{33}$ an $\mathrm{O}_{2}$ mass fraction of $0.5 \%$ would be expected. The Schmidt number has significant influence on the mass fractions in the throat (e.g. oxygen). The increasing combustion efficiency explains the increased pressure for lower Schmidt numbers. This study shows that the effects of Prandtl and Schmidt number are significant. 

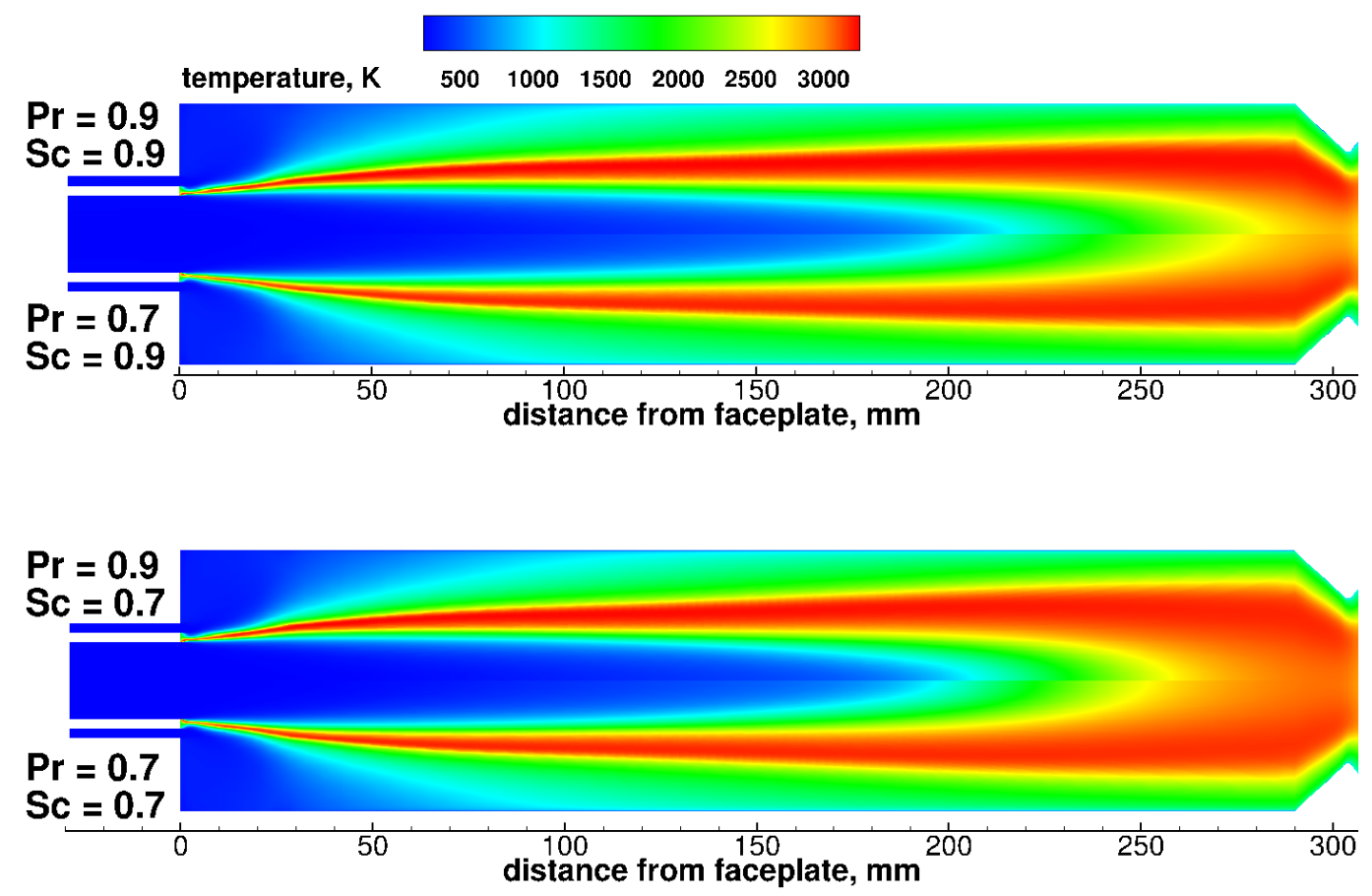

Figure 8. Temperature contours for different turbulent Prandtl and Schmidt numbers for the q- $\omega$ turbulence model.

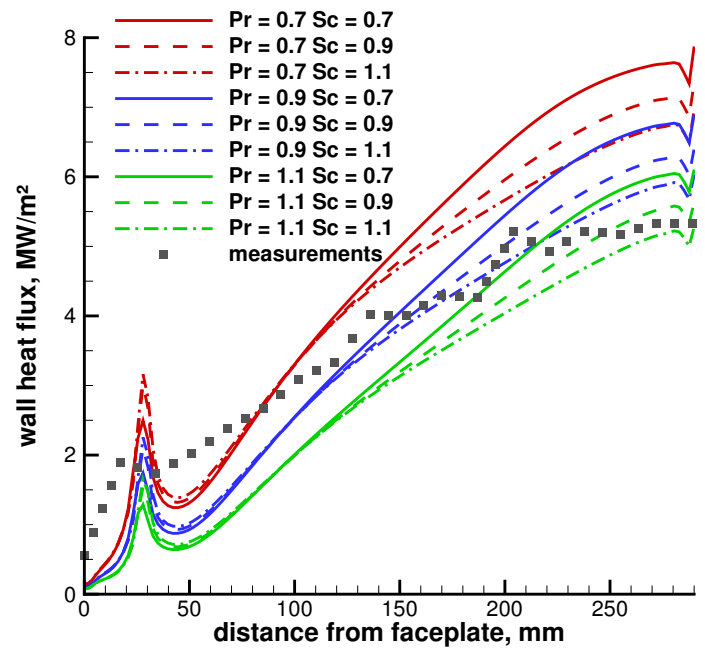

(a) Wall heat flux.

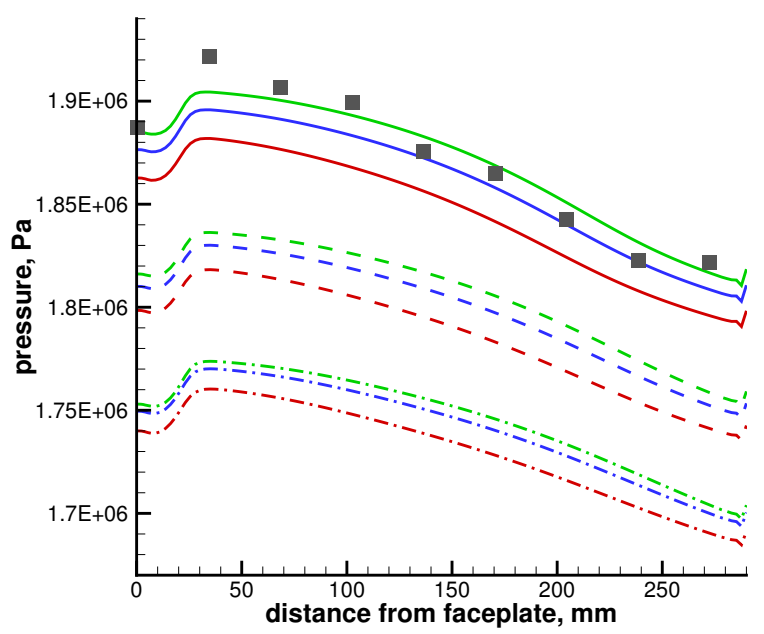

(b) Pressure.

Figure 9. Wall heat flux (left) and pressure (right) profiles along the $x$-axis for different turbulent Prandtl and Schmidt numbers using the $q-\omega$ turbulence model. 


\begin{tabular}{l|lllllllll}
$\operatorname{Pr}_{t} / S c_{t}$ & $0.7 / 0.7$ & $0.7 / 0.9$ & $0.7 / 1.1$ & $0.9 / 0.7$ & $0.9 / 0.9$ & $0.9 / 1.1$ & $1.1 / 0.7$ & $1.1 / 0.9$ & $1.1 / 1.1$ \\
\hline$Y_{\mathrm{O}_{2}}[\%]$ & 4.85 & 9.37 & 13.62 & 4.86 & 9.51 & 13.93 & 4.97 & 9.77 & 14.38 \\
$Y_{\mathrm{CH}_{4}}[\%]$ & 0.92 & 2.03 & 3.18 & 0.86 & 2.01 & 3.23 & 0.83 & 2.00 & 3.28 \\
$Y_{\mathrm{H}_{2} \mathrm{O}}[\%]$ & 35.92 & 33.29 & 31.32 & 35.64 & 32.83 & 30.74 & 35.32 & 32.37 & 30.16 \\
$Y_{\mathrm{CO}_{2}}[\%]$ & 18.88 & 18.96 & 18.75 & 18.38 & 18.36 & 17.99 & 18.02 & 17.91 & 17.39 \\
$P_{\text {max }}[$ bar $]$ & 18.81 & 18.18 & 17.60 & 18.96 & 18.30 & 17.70 & 19.04 & 18.36 & 17.74
\end{tabular}

Table 1. Mass flow-weighted mass fractions at the throat.

\section{V.D. Three-dimensional RANS simulation}

Figure 10 shows the temperature contours in $z$-, diagonal, and $x$-plane. Please note that the contour plots are not to scale but strongly stretched in $y$ - and $z$-direction. The axial temperature contour in the $z$-plane is similar to the contours of the axisymmetric simulation (see Fig. 6). A major difference is the higher temperature near the wall at the end of the chamber and in the nozzle.

The temperature contours along $x$-planes illustrate the three-dimensional nature of this chamber. The flame is circular and undisturbed by the chamber walls in the first half of the chamber. In the last two $x$-planes $(220 \mathrm{~mm}$ and $290 \mathrm{~mm}$ ) a strong deviation from the initial round shape is visible. This shows that the axisymmetric assumption for the flame structure is at most adequate at the beginning of the chamber. Due to the different temperature distribution close to the wall, strong variations of the wall heat flux in the circumferential direction are expected.

The wall heat flux profiles in axial direction are shown in Fig. 11 a). For comparison with the experimental values, three different profiles are plotted: the heat flux at the center line, the heat flux at the corner, and the heat flux averaged over the entire surface. These wall heat fluxes vary significantly. The wall heat fluxes for the two-dimensional simulations with different diameters and the experimental values are depicted as well. As can be seen, the center line heat flux vastly overpredicts the measured values, whereas the heat flux predictions in the corners are much lower. The averaged values compare quite well with the measured values. As discussed in Sec. V.A the three different diameters for the axisymmetric simulation each preserve a unique feature of the square original chamber. There is a strong resemblance for each two-dimensional wall heat flux with one of the three-dimensional wall heat fluxes. As expected, the wall heat flux distribution of the $2 \mathrm{D}$ simulation with the smallest diameter $(\mathrm{D}=12 \mathrm{~mm})$ is comparable with the center line distribution. The corner wall heat flux profile is similar to the one from the axisymmetric simulation with $\mathrm{D}=17 \mathrm{~mm}$. Whereas the averaged wall heat flux profile is similar to the simulation with a diameter of $13.5 \mathrm{~mm}$. Please note that the 2D wall heat fluxes are not scaled with their respective surface areas.

In Fig 11 b) the circumferential distribution at different axial positions is shown. The absolute differences between the corner and the symmetry wall heat fluxes increase with the distance from the faceplate. The heat fluxes in the center of the combustion chamber (experimental measurement position) can be more than
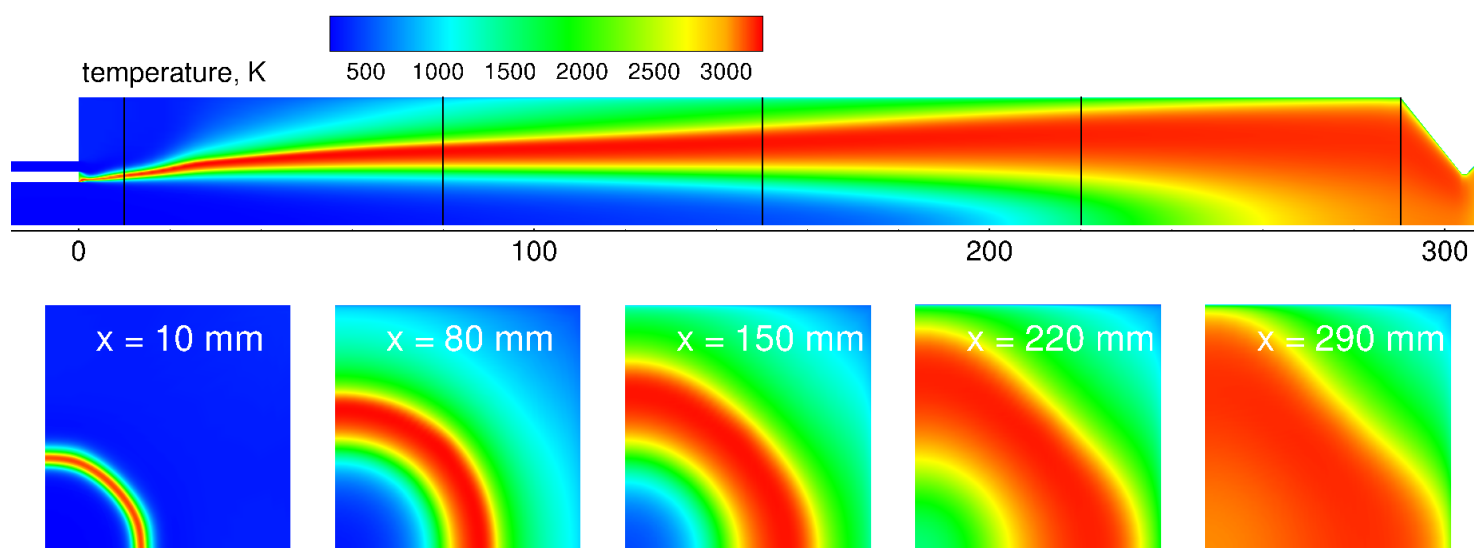

Figure 10. Temperature contours for the three-dimensional simulation on the $z$-plane (top) and different $x$-planes (bottom). 


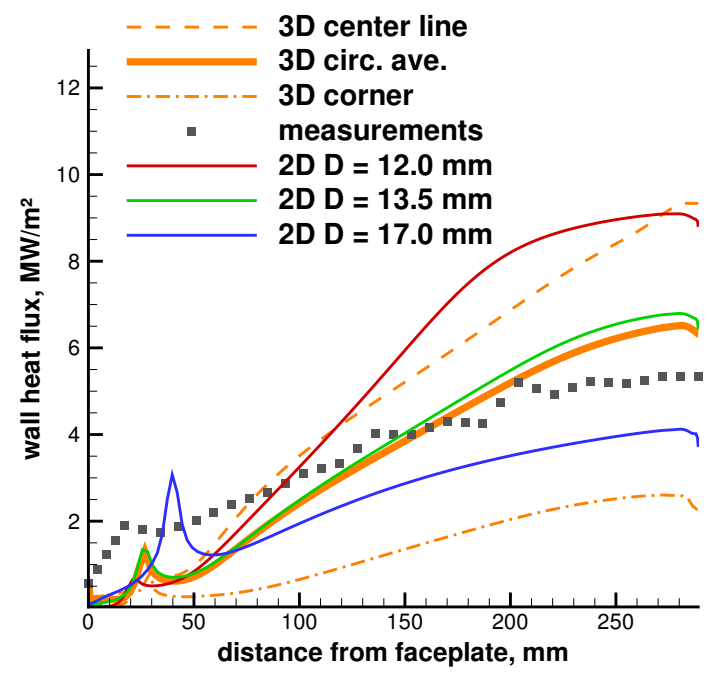

(a) Wall heat flux profiles in axial direction.

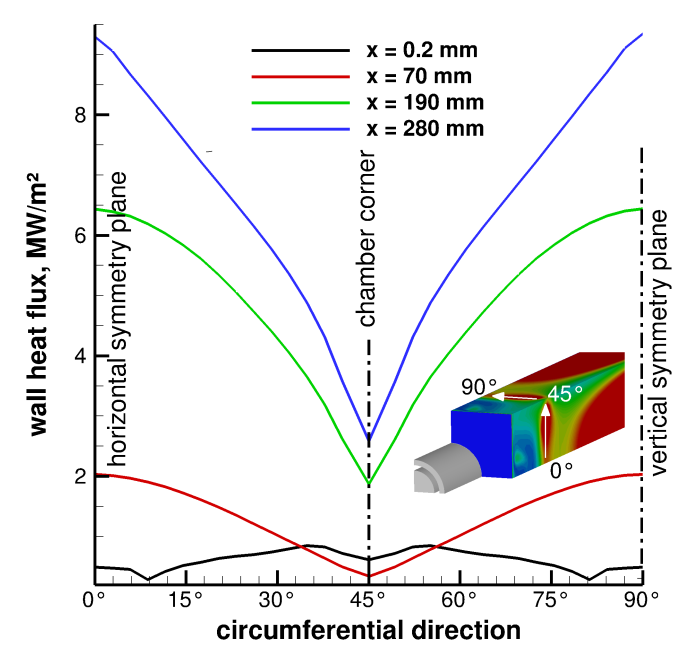

(b) Wall heat flux profiles in circumferential direction.

Figure 11. Wall heat flux distribution for the three-dimensional simulation.

three times larger than in the corners. These strong differences in the wall heat flux distribution question the assumption of a constant wall temperature in circumferential directions during the setup of the simulation. It is also not clear whether the experimental wall heat fluxes should be compared to the central line wall heat flux, the averaged wall heat flux, or even a combination of both.

Figure 12 shows the pressure distribution along the axial direction for the three-dimensional simulation compared with values from the two-dimensional simulation $(\mathrm{D}=13.5 \mathrm{~mm})$ and the experiment. The pressure is underpredicted by the three-dimensional simulation compared to the experimental values and the twodimensional simulation. Although the averaged pressure is lower, the pressure profile shows the same trend as in the two-dimensional simulation.

\section{V.D.1. Recirculation zone}

In axisymmetric chambers usually a single surrounding recirculation zone is present. ${ }^{10}$ The rectangular chambers' recirculation zones have a different structure. ${ }^{11}$ Figure 13 visualizes the recirculation zone via stream traces in two different planes. Figure 13 a) shows the symmetry plane $(\mathrm{z}=0)$ and Fig. $13 \mathrm{~b})$ shows the diagonal plane. The isoline for zero axial velocity is drawn in purple and marks the center of the recirculation

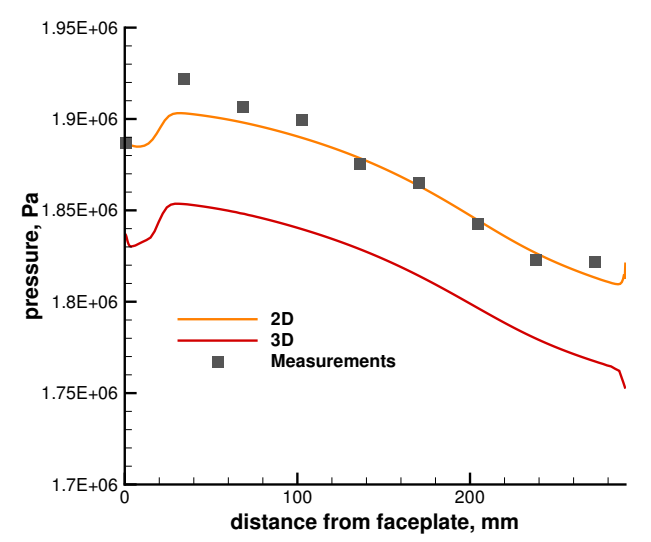

Figure 12. Pressure distribution for the three-dimensional simulation. 


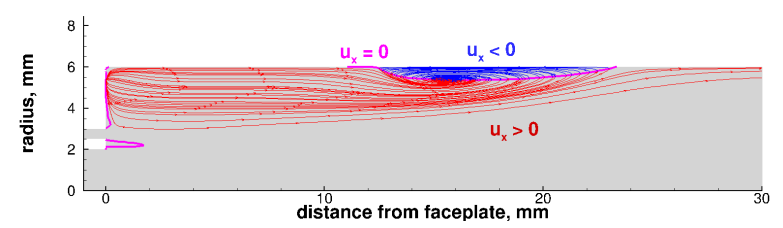

(a) Symmetry plane.

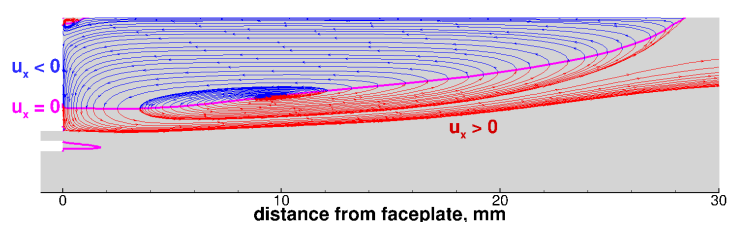

(b) Diagonal plane.

Figure 13. 2D presentation of the stream traces of recirculation zones for the 3D RANS simulation in the $z$-plane (left) and the diagonal plane (right). The zero axial velocity iso-contour is indicated with a purple line.

zone which is "rotating around it". The stream lines are colored with blue for negative and red for positive axial velocities. In the symmetry plane only a small recirculation zone away from the faceplate (from $12 \mathrm{~mm}$ to $23 \mathrm{~mm}$ ) is visible. The diagonal plane has a large recirculation zone extending from the faceplate to 28 $\mathrm{mm}$ into the chamber.

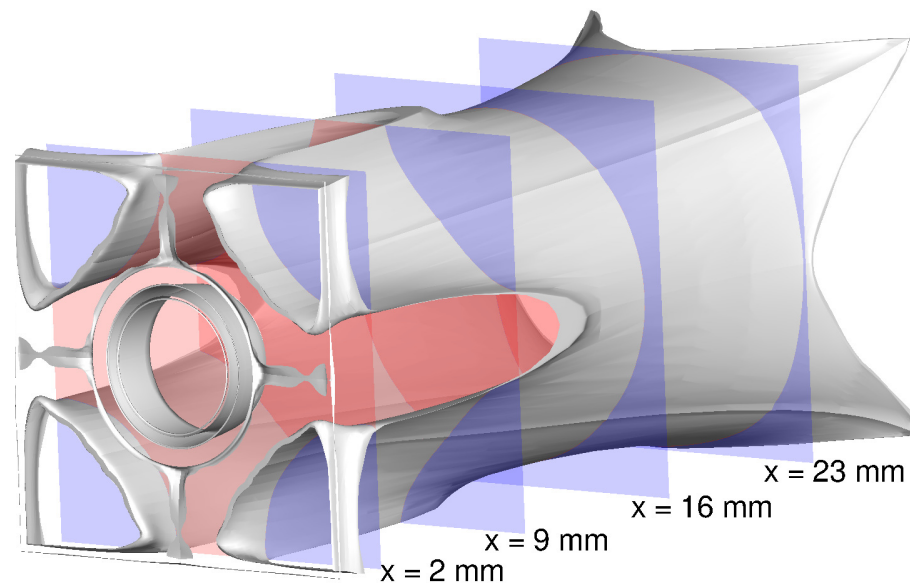

Figure 14. Visualization of the shape of the three-dimensional recirculation zone with an zero velocity iso-contour. Colors blue and red indicate negative and positive axial velocities.

To get a better understanding of the 3D structure of the recirculation zone, Fig. 14 illustrates it in a three-dimensional plot. The structure is depicted by $x$-slices through the recirculation zone and a zero axial velocity iso-contour. Colors blue and red indicate negative and positive axial velocities. Near the faceplate, the four separate recirculation zones in each corner of the chamber are visible by vanishing negative velocities. In the diagonal plane of the chamber, the recirculation zone has the usual structure with a negative velocity at the wall as shown in Fig. $13 \mathrm{~b}$ ). From the structure of the iso-contours in the corner it is apparent why no recirculation zone near the faceplate is visible in Fig. 13 a). Further downstream of the faceplate, the four separate recirculation zones merge into a single surrounding zone. This is indicated by the constant negative velocity near the wall in the third slice as well as the continuous iso-contour. The recirculation zone is much thicker and longer in the corners. Up to now, it is still possible that this observed structure is due to error introduced by the symmetry boundary conditions, since all other simulations reporting such a structure were also using symmetry boundary conditions.

The simulation shows significant three-dimensional effects occurring in this rocket combustion chamber. It is assumed that by neglecting them, significant errors are introduced. Surprisingly, no better comparison with the experimental data could be achieved with the three-dimensional simulation.

\section{Conclusion and future work}

A square cross-section methane combustion model chamber has been successfully simulated. A threedimensional and simplified axisymmetric steady-state simulations were presented. Influence factors have been investigated using a simplified setup and a significant impact of the turbulent Prandtl and Schmidt numbers on the solution was established. The three-dimensional simulation showed strong differences in the 
flow structure compared to the simplified axisymmetric simulation. But their influence on the wall heat flux and pressure profile is small. The quantitative agreement with the experimental data is good. Nevertheless, the qualitative agreement shows some shortcomings, especially for the heat flux near the faceplate and the pressure at the end of the chamber. These shortcomings are attributed to an incorrect flame position and an improvement with unsteady simulations is expected. Future work will include investigations into the lower pressure level of the three-dimensional simulation.

\section{Acknowledgments}

We wish to thank the German Research Foundation (Deutsche Forschungsgemeinschaft, DFG) for financial support in the framework of the Sonderforschungsbereich/Transregio 40 and the research group of Professor Haidn (IFA, TU München). Computational resources have been provided by the High Performance Computing Center Stuttgart (HLRS).

\section{References}

${ }^{1}$ Marshall, W. M., Pal, S., Woodward, R. D., and Santoro, R. J., "Benchmark wall heat flux data for a $\mathrm{GO}_{2} / \mathrm{GH}_{2}$ single

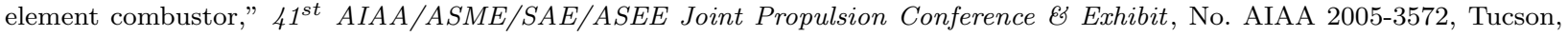
Arizona, 2005.

${ }^{2}$ Grisch, F., Bouchardy, P., Vingert, L., Clauss, W., Oschwald, M., Stel'mack, O. M., and Smirnov, V. V., CARS Measurements at High Pressure in Cryogenic LOX/GH2 Jet Flames, Vol. 200 of Progress in Astronautics and Aeronautics, chap. 10, American Institute of Aeronautics and Astronautics, Reston,VA,USA, 2004, pp. 369-404.

${ }^{3}$ Locke, J. M., Pal, S., Woodward, R. D., and Santoro, R. J., "Toward Time-Resolved measurements in a Gaseous Hydrogen/Oxygen Rocket," 45th AIAA/ASME/SAE/ASEE Joint Propulsion Conference ES Exhibit, No. AIAA 2009-5395, Denver, Colorado, 2009.

${ }^{4}$ Pal, S., Marshall, W., Woodward, R., and Santoro, R., "Wall heat flux measurements for a uni-element $\mathrm{GO}_{2} / \mathrm{GH}_{2}$ shear coaxial injector," Third International Workshop on Rocket Combustion Modeling, Paris,France, 2006, pp. 13-15.

${ }^{5}$ Locke, J. M., Pal, S., and Woodward, R. D., "Chamber Wall Heat Flux Measurements for a $\mathrm{LOX} / \mathrm{CH}_{4} \mathrm{Uni}_{-} \mathrm{element}$ Rocket," 43 ${ }^{\text {rd }}$ AIAA/ASME/SAE/ASEE Joint Propulsion Conference 8 Exhibit, No. AIAA 2007-5547, Cincinnati, OH., 2007.

6 "TNF Workshop," Online; accessed April 9th 2012, http://www.sandia.gov/TNF/abstract.html.

${ }^{7}$ Tucker, P. K., Menon, S., Merkle, C. L., Oefelein, J. C., and Yang, V., "An approach to improved credibility of CFD simulations for rocket injector design," $43^{\text {rd }}$ AIAA/ASME/SAE/ASEE Joint Propulsion Conference Es Exhibit, No. AIAA 2007-5572, Cincinnati, OH., 2007.

${ }^{8}$ Ivancic, B., Riedmann, H., Frey, M., Knab, O., Karl, S., and Hannemann, K., "Investigation of different modeling approaches for CFD simulation of high pressure rocket combustors," $5^{\text {th }}$ European Conference for Aeronautics and Space Sciences (EUCASS), Munich, Germany, 2013.

${ }^{9}$ Riedmann, H., Kniesner, B., Frey, M., and Munz, C.-D., "Modeling of combustion and flow in a single element $\mathrm{GH}_{2} / \mathrm{GO}_{2}$ combustor," CEAS Space Journal, Vol. 6, No. 1, 2014, pp. 47-59.

${ }^{10}$ Lempke, M., Keller, R., and Gerlinger, P., "Influence of Spatial Discretization and Unsteadiness on the Simulation of Rocket Combustors," International Journal for Numerical Methods in Fluids, Vol. 79, No. 9, 2015, pp. 437-455.

${ }^{11}$ Lempke, M., Gerlinger, P., Seidl, M. J., and Aigner, M., "Unsteady High-Order Simulation of a Liquid Oxygen/Gaseous Hydrogen Rocket Combustor," Journal of Propulsion and Power, Vol. 31, No. 6, 2015, pp. 1715-1726.

${ }^{12}$ Lian, C. and Merkle, C. L., "Contrast between steady and time-averaged unsteady combustion simulations," Computers Es Fluids, Vol. 44, No. 1, 2011, pp. 328-338.

${ }^{13}$ Gerlinger, P., Stoll, P., and Brüggemann, D., "An Implicit Multigrid Method for the Simulation of Chemically Reacting Flows," Journal of Computational Physics, Vol. 146, 1998, pp. 322-345.

${ }^{14}$ Gerlinger, P., Möbus, H., and Brüggemann, D., "An implicit multigrid method for turbulent combustion," Journal of Computational Physics, Vol. 167, No. 2, 2001, pp. 247-276.

${ }^{15}$ Gerlinger, P., "Investigation of an assumed PDF approach for finite-rate chemistry," Combustion Science and Technology, Vol. 175, No. 5, 2003, pp. 841-872.

${ }^{16}$ Gerlinger, P., Stoll, P., Kindler, M., Schneider, F., and Aigner, M., "Numerical investigation of mixing and combustion enhancement in supersonic combustors by strut induced streamwise vorticity," Aerospace Science and Technology, Vol. 12, No. 2, Mar 2008, pp. 159-168.

${ }^{17}$ Gerlinger, P., Nold, K., and Aigner, M., "Influence of reaction mechanisms, grid spacing, and inflow conditions on the numerical simulation of lifted supersonic flames," International Journal for Numerical Methods in Fluids, Vol. 62, No. 12, 2010, pp. $1357-1380$.

${ }^{18}$ Gerlinger, P. and Brüggemann, D., "Multigrid Convergence Acceleration for Turbulent Supersonic Flows," International Journal for Numerical Methods in Fluids, Vol. 24, 1997, pp. 1019-1035.

${ }^{19}$ Gerlinger, P. and Brüggemann, D., "An Implicit Multigrid Scheme for the Compressible Navier-Stokes Equations with Low-Reynolds-Number Turbulence Closure," Journal of Fluids Engineering, Vol. 120, 1998, pp. 257-262.

${ }^{20}$ Gerlinger, P., Noll, B., and Aigner, M., "Assumed PDF modeling and PDF structure investigation using finite-rate chemistry," Progress in Computational Fluid Dynamics, an International Journal, Vol. 5, No. 6, 2005, pp. 334-344. 
${ }^{21}$ Gerlinger, P., "Multi-dimensional limiting for high-order schemes including turbulence and combustion," Journal of Computational Physics, Vol. 231, No. 5, 2012, pp. 2199-2228.

${ }^{22}$ Coakley, T. and Huang, P., "Turbulence Modeling for High Speed Flows," AIAA Paper, 1992-0436, 1992.

${ }^{23}$ Menter, F. R., "Two-equation eddy-viscosity turbulence models for engineering applications," AIAA Journal, Vol. 32, No. 8, 1994, pp. 1598-1605.

${ }^{24}$ Jameson, A. and Yoon, S., "Lower-upper implicit schemes with multiple grids for the Euler equations," AIAA Journal, Vol. 25, No. 7, 1987, pp. 929-935.

${ }^{25}$ Shuen, J.-S., "Upwind differencing and LU factorization for chemical non-equilibrium navier-stokes equations," Journal of Computational Physics, Vol. 99, No. 2, 1992, pp. 233-250.

${ }^{26}$ Keller, R., Lempke, M., Simsont, Y. H., Gerlinger, P., and Aigner, M., "Parallelization and Performance Analysis of an Implicit Compressible Combustion Code for Aerospace Applications," High Performance Computing in Science and Engineering'14, Springer, Heidelberg,Germany, 2015, pp. 251-266.

${ }^{27}$ Liou, M.-S., "A sequel to AUSM, Part II : AUSM+ ${ }^{+}$-up for all speeds," Journal of Computational Physics, Vol. 214, No. 1, 2006, pp. 137-170.

${ }^{28}$ Slavinskaya, N. A., Abbasi, M., Weinschenk, M., and Haidn, O. J., "Methane Skeletal Mechanism for Space Propulsion Applications," 5th International Workshop on Model Reduction in Reacting Flows (IWMRRF), Springer, Spreewald, Germany, 2015, pp. 36-37.

${ }^{29}$ Celano, M. P., Silvestri, S., Schlieben, G., Kirchberger, C., and Haidn, O. J., "Injector Characterization for a GOX-GCH4 Single Element Combustion Chamber," $5^{\text {th }}$ European Conference for Aeronautics and Space Sciences (EUCASS), Munich, Germany, 2013.

${ }^{30}$ Celano, M., Silvestri, S., Schlieben, G., Kirchberger, C., Haidn, O. J., Dawson, T., Ranjan, R., and Menon, S., "Experimental and Numerical Investigation for a GOX-GCH4 Shear-Coaxial Injector Element," 2014 Space Propulsion Conference, May 2014, No. SP-2014-2969417, Cologne, Germany, 2014.

${ }^{31}$ Tucker, P. K., Menon, S., Merkle, C. L., Oefelein, J. C., and Yang, V., "Validation of high-fidelity CFD simulations for rocket injector design," $44^{\text {th }}$ AIAA/ASME/SAE/ASEE Joint Propulsion Conference ES Exhibit, No. AIAA 2008-5226, Hartford,CT, 2008.

${ }^{32}$ Masquelet, M. and Menon, S., "Large-Eddy Simulation of Flame-Turbulence Interactions in a Shear Coaxial Injector," Journal of Propulsion and Power, Vol. 26, No. 5, 2010, pp. 924-935.

${ }^{33}$ Gordon, S. and McBride, B. J., Computer program for calculation of complex chemical equilibrium compositions and applications, National Aeronautics and Space Administration, Office of Management, Scientific and Technical Information Program, 1996. 